\title{
Design of a Sapling Branch Grafting Robot
}

\author{
Qun Sun, ${ }^{1}$ Dongjie Zhao, ${ }^{1,2}$ Chong Wang, ${ }^{1}$ and Ying Zhao ${ }^{1}$ \\ ${ }^{1}$ School of Mechanical \& Automotive Engineering, Liaocheng University, Liaocheng 252059, China \\ ${ }^{2}$ College of Engineering, China Agricultural University, Beijing 100083, China
}

Correspondence should be addressed to Qun Sun; sunxiaoqun97@163.com

Received 4 July 2014; Revised 11 October 2014; Accepted 15 October 2014; Published 23 November 2014

Academic Editor: Giovanni Muscato

Copyright (C) 2014 Qun Sun et al. This is an open access article distributed under the Creative Commons Attribution License, which permits unrestricted use, distribution, and reproduction in any medium, provided the original work is properly cited.

\begin{abstract}
The automatic sapling grafting methods and grafting robot technologies are not comprehensively studied despite the fact that they are urgently required in practice. For this reason, a sapling grafting robot is developed to implement automatic grafting for saplings. The developed grafting robot includes clipping mechanism, moving mechanism, cutting mechanism, binding mechanism, and Arduino MCU based control system, which is capable of clipping, moving, positioning, cutting, grafting, and binding saplings. Experiments show that the stock cutting efficiency is $98.4 \%$, the scion cutting efficiency is $98.9 \%$, the grafting efficiency is $87.3 \%$, and the binding efficiency is $68.9 \%$.
\end{abstract}

\section{Introduction}

Sapling grafting is an ancient technology that can assist breeding of good plant species, enhance their ability of antiillness, improve the fruit quality, and strengthen their adaptabilities to harsh environments. The earliest literatures about manual citrus sapling grafting techniques appeared around 3000 years ago in China.

Japan is the first country in the world that developed vegetable grafting robots. The first commercial model of a grafting robot (GR800 series, Iseki \& Co. Ltd., Matsuyama, Japan) became available for cucurbits back in 1993 [1-3]. Since then, semiautomated or fully automated vegetable grafting robots have been invented by several agricultural machine companies, and several commercial models became available in East Asia, Europe, and more recently the U.S. [4-9].

However, grafting robots for saplings have not been comprehensively studied. A semiautomatic grape grafting robot was developed by CFCL in France to improve the grape production. Till present, the sapling grafting works are still following traditional manual methods in China, which are labour intensive but less productive. Besides, the grafted saplings often exhibit low survival rates and are less adaptive to the seasonal planting characteristics, which made it difficult to achieve industrialized sapling breeding production.
Some similar literatures about sapling grafting robot could be found in China in the following areas.

(1) Sapling Force Characteristics Experiments. Li et al. studied peach saplings and carried out experiments on peach force characteristics such as the cutting resistance force in vertical, traverse, and inclined cutting manners and also studied the variations of compressive injury forces [10]. Zhao et al. investigated poplar saplings based on a stick-to-stick grafting manner and studied the varying relationships between cutting resistance force and cutting depth, as well as the impact of scion diameter on the cutting resistance force [11].

(2) Sapling Grafting Mechanism Design. Li et al. designed a cutting mechanism to carry out experiments for peach sapling grafting, and the average cutting successful rate of this mechanism reached up to $99.14 \%$ [12]. Zhao et al. created a scion cutting mechanism for the poplar sapling studies, which provided a successful rate of $96.5 \%$ [13]. Jiang designed a pure mechanical grape sapling mechanism for a stick-to-stick grafting approach. Its grafting efficiency is 15-20 times that of manual work and the grafting survival rate exceeded $85 \%$ [14].

The Harbin Forestry Machinery Institute of China's Forestry Administration Bureau developed a camellia grafting robot based on a cutting grafting method [15]. The author of this paper and his research team also initially studied 


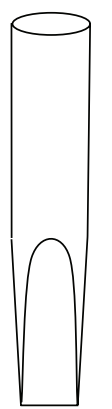

(a)

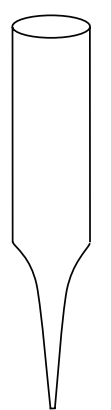

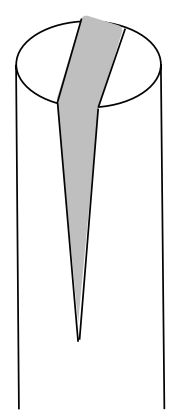

(b)

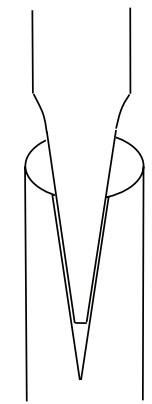

(c)
FIGURE 1: Operation flow of sapling cleft grafting by hand.

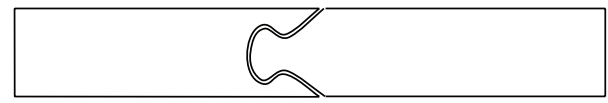

Figure 2: “ $\Omega$ ” shaped blades.

cleft grafting mechanism for poplar saplings and associated automatic grafting devices [16].

This study developed a sapling grafting robot and carried out experiments for hard branch grafting applications, in order to investigate universal methods for grafting hard sapling branches and the associated grafting robot devices.

\section{Sapling Hard Branch Grafting Method}

2.1. Grafting Method. Cleft grafting is the most important method for sapling hard branch grafting. The manual operation flow diagram is shown in Figure 1. Firstly, a scion branch is cut into a double wedge shaped incision and a rootstock is cut into an "I" shaped cleft; then, force the cross section of the rootstock cleft to be tightly attached to the wound surface of the scion wedge incision, leaving no gap on the top and the bottom, and if loose further bind tightly.

In order to accurately align the cutting surfaces of the scion and the rootstock cross sections and ensure a tight binding of the saplings, people now use " $\Omega$ " shaped blades to directly cut scions and rootstocks, as shown in Figure 2, which simplifies the program of cleft grafting process and enhances grafting efficiency. Therefore, the " $\Omega$ " shaped blades grafting method is adopted in the paper.

\subsection{Sapling Grafting Mechanical Characteristics Analysis. By} a mechanical cold cutting manner, the sapling mechanical grafting characteristics include scion and rootstock growing geometry parameters, cutting force characteristics, cutting surface physiology, and biochemistry characteristics. In particular, the scion and rootstock cutting force characteristics are directly associated with the design of the grafting robot mechanism variables [11]. Using force measurement equipment, the relationship between cutting force and cutting depth could be obtained by 100 branch samples, as shown in Figure 3. Some key parameters are also confirmed using sensors as in the following chart [11].

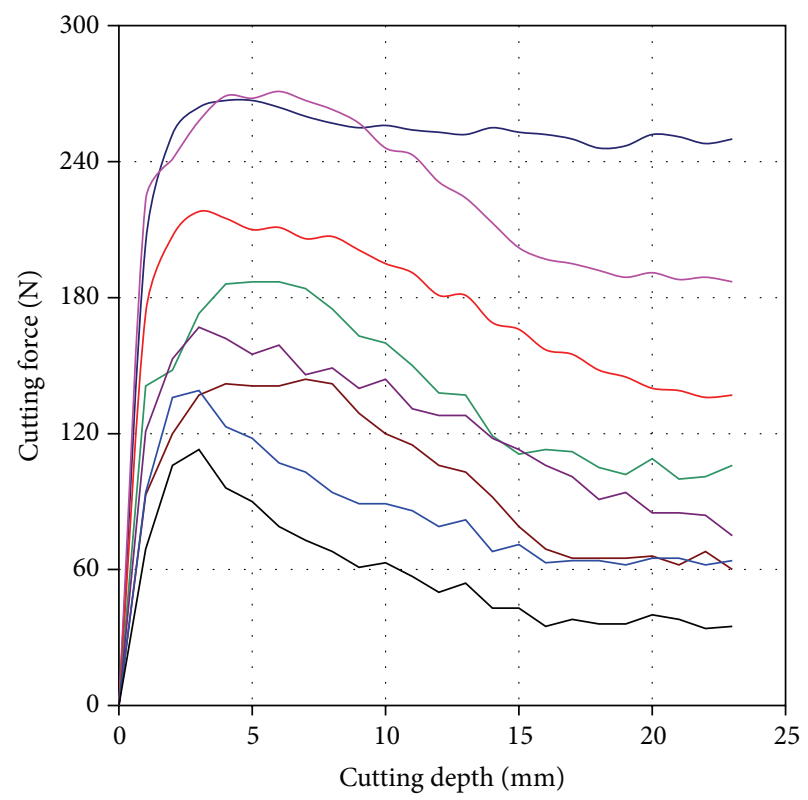

FIGURE 3: Relationship between cutting force and cutting depth.

\section{Design of Mechanical System for Sapling Grafting Robot}

\subsection{Design of Mechanical System for \\ Integrated Sapling Grafting Robot}

3.1.1. Sapling Grafting Robot Working Process. The working process of a sapling grafting robot is as follows: feeding scion and rootstock saplings $\rightarrow$ moving saplings $\rightarrow$ cutting scions and rootstocks $\rightarrow$ moving saplings $\rightarrow$ attaching saplings $\rightarrow$ binding saplings $\rightarrow$ detaching saplings. Among these, feeding and finally detaching scion and rootstock saplings need manual operation. The working process of the sapling grafting robot is shown in flow diagram in Figure 4.

3.1.2. Integrated Structure of the Sapling Grafting Robot. The overall structure of the sapling grafting robot consists of a frame, a robot hand to handle rootstock, another robot hand to clamp scion, rootstock moving mechanism, scion moving mechanism, rootstock cutting mechanism, scion cutting mechanism, and binding mechanism. It adopts modular design concepts so that the functionalities are relatively independent of each other and utilizes many standard parts and mature products such as standard aluminum frame, angle parts, robot hands, and grafting blades. The overall mechanical structure of the example robot machine is shown in Figure 5.

\subsection{Design of the Key Structures of the Sapling Grafting Robot}

3.2.1. Scion and Rootstock Clipping Robot Hands. The scion and rootstock clipping robot hands are of the same structures; both include screw driver and motor, guiding shaft, linear bearing, robot hand, and other parts, as shown in Figure 6. 


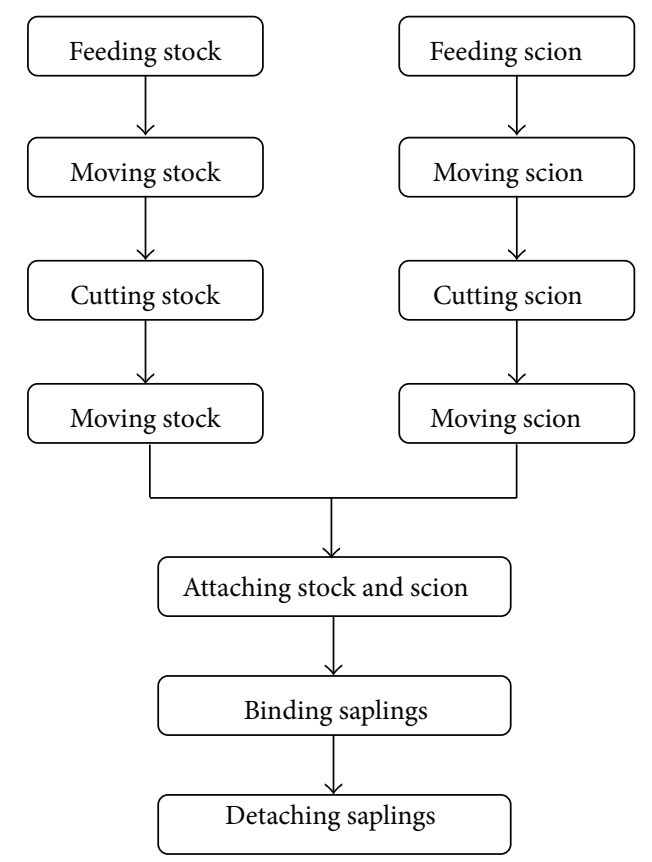

Figure 4: Work flow of grafting robot.

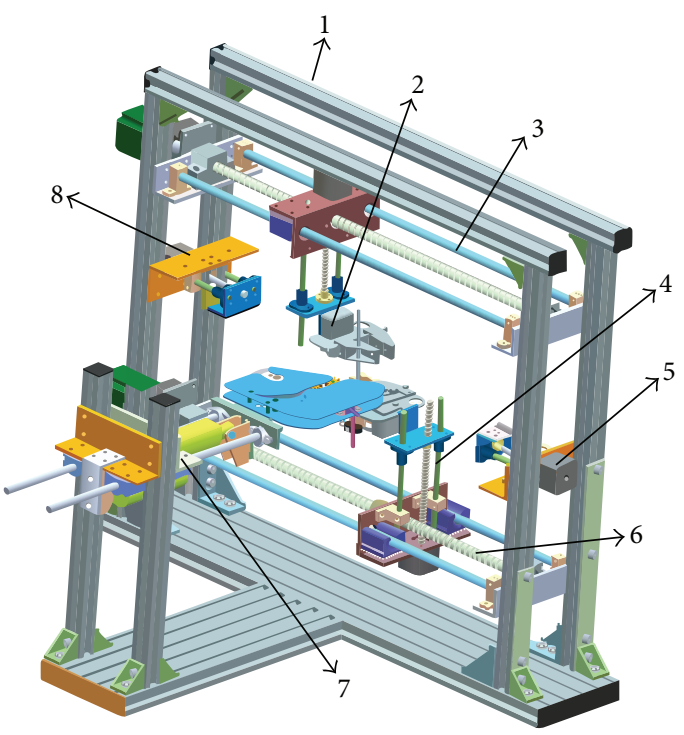

FIGURE 5: The whole mechanical structure of grafting robot. 1: frame, 2: scion clipping robot hand, 3: scion moving mechanism, 4: stock clipping robot hand, 5: stock cutting mechanism, 6: scion moving mechanism, 7: binding mechanism, and 8: scion cutting mechanism.

The robot hand is based on BigHand single steering clipping actuator, which can control clipping and releasing rootstock and scion as well as adjusting the clipping force. The screw motor adopts a BG42 step motor that can control the robot hand to bring scion and rootstock to move vertically along the bare shaft.

3.2.2. Rootstock and Scion Moving Mechanism. The moving mechanisms for rootstocks and scions have basically the same

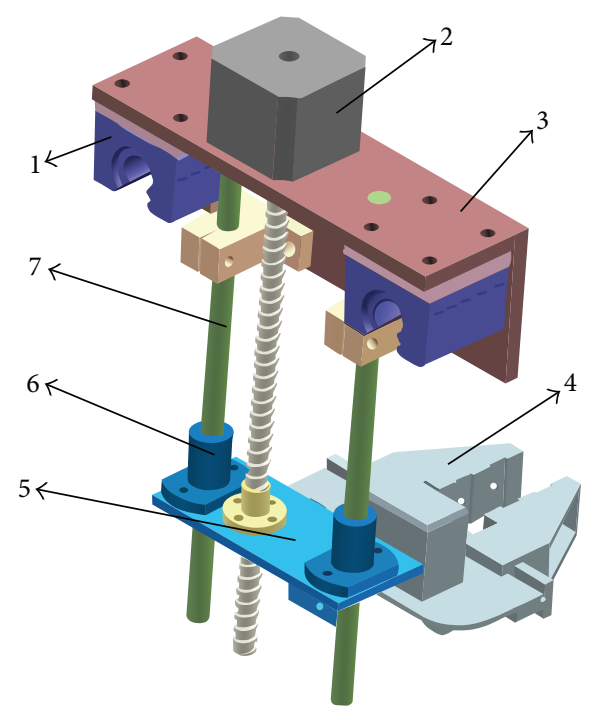

Figure 6: Structure of the clipping mechanism. 1: sliding unit, 2: screw motor, 3: connection base, 4: robot hand, 5: mounting base for robot hand, 6: linear bearing, and 7: bare guiding shaft.

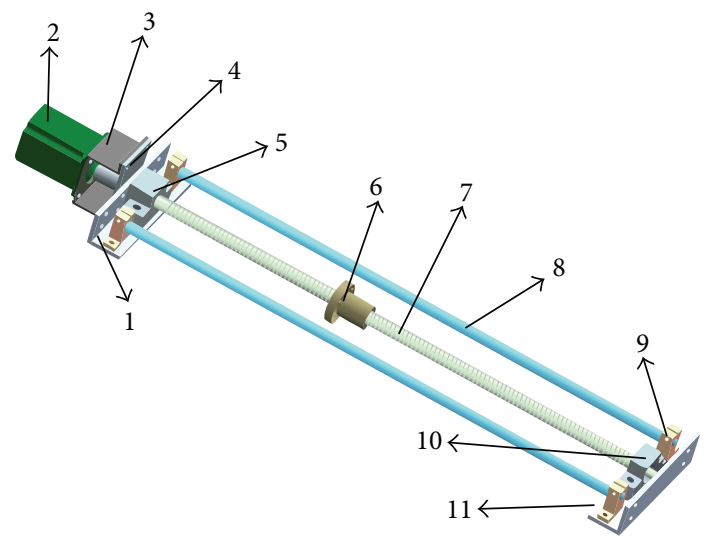

FIgURE 7: Structure of the moving mechanism. 1: left supporting base, 2: driving motor, 3: motor mounting base, 4: motor connection board, 5: front screw mounting base, 6 : driving screw nut, 7: driving screw, 8: bare guiding shaft, 9: bare supporting base, 10: rear screw mounting base, and 11: right supporting base.

structures. They both include supporting base, transmission motor, screw, and nut as shown in Figure 7.

The driving motor uses a 57BYG250-76 step motor, which powers the screw to rotate through a coupler and drives the clipping robot hand connected on the screw nut to move horizontally along the bare shaft.

3.2.3. Rootstock and Scion Cutting Mechanism. The scion and rootstock cutting mechanisms have the same structure as they both include screw motor, bare guiding shaft, screw nut, and cutting parts, as shown in Figure 8.

The screw motor drives the cutting blade to move horizontally through connection on the blade mounting base and the mounting board, so as to cut the saplings allocated between the cutting blade and the rubber pad. The cutting 


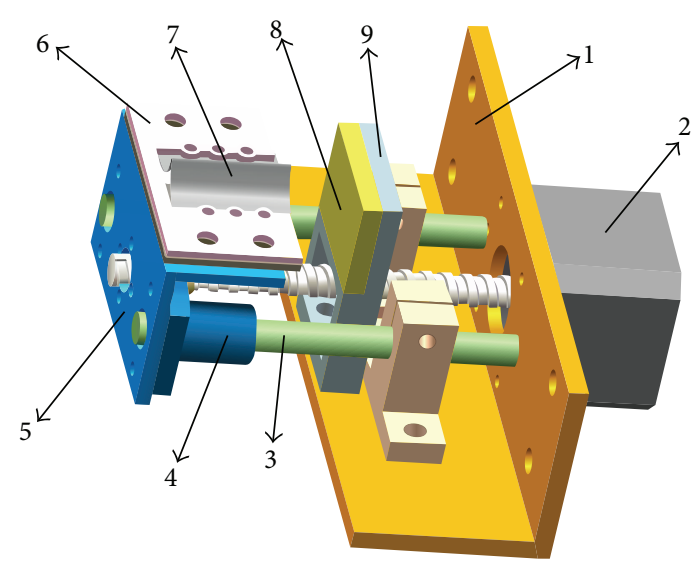

FIGURE 8: Structure of cutting mechanism. 1: mounting base, 2: screw motor, 3: bare guiding shaft, 4: linear bearing, 5: cutting blade mounting base, 6 : cutting blade mounting board, 7: cutting blade, 8 : rubber pad, and 9: rubber pad back base.

blade is a " $\Omega$ " shaped knife as extensively adopted in manual grafting tools.

The flow diagram of the cutting force test experiments is shown in Figure 9. Two FSR402 film pressure sensors are fixed on a YANSHEN hand-held sapling grafting device, forming half-bridge circuit through a bridge circuit junction box. The signal goes through a resistance strain gauge, a data acquisition device and then reaches the PC. Then, Vib'SYS signal gathering, processing, and analyzing system was employed for processing. That testing device was used to measure the cutting forces over 100 poplar branches and grape branches each of $6 \mathrm{~mm}$ thickness. The measured results indicated that, using the " $\Omega$ " shape blades, the required cutting forces for poplar branches are less than $300 \mathrm{~N}$. from

The required power of the driving motor is calculated

$$
P_{m}=\lambda P=\lambda \frac{T n}{9550}=\frac{\lambda F p \eta \cdot(\pi / 2) \cdot n}{9550},
$$

where $F$ is the required cutting force, $\lambda$ is the surplus coefficient, $T$ is the motor torque, $n$ is the screw rotational speed, $p$ is the screw lead, and $\eta$ is the screw efficiency.

According to the calculation, the adopted screw motor is Shidai Chaoqun type BG42 step motor.

3.2.4. Sapling Binding Mechanism. The binding mechanism includes base components, moving parts, and binding components. The structure is shown in Figure 10.

By adjusting the height of the moving components of this binding device, the long slot of the binding components can be aligned with the sapling intake nozzle. The binding belt should be set around the gear and locked between the gear and the claw. The motor drives the pusher to move which sends the sapling into the tooth space of the gear wheel and then moves along the long slot. The binding belt wraps the sapling end and bonds tightly under the pressure from the claw. Then, the electromagnet drives the cutting blade to cut off the binding belt. The working process is as shown in Figure 10(c).

\section{Design of Sapling Robot Control System}

4.1. Design of Overall Control System. The control objects include step motor, servo motor, LCD display, DC motor, and electromagnet, whilst the measured objects include micro switch signal and approach switch signal. The control system diagram is shown in Figure 11.

4.2. Design of Control System Hardware. The controller adopts Arduino MCU and the hardware design includes the following.

(1) Front Channel Configurations. The sensors of the control system are 2 micro switches and 2 approach switches; both include TTL levels. The front channel configuration includes 4-way binary input circuit.

(2) Rear Channel Configurations. The control system requires 2 servo motors, 6 step motors, 1 DC motor, 1 electromagnet, and $1 \mathrm{LCD}$ module. According to modular design concepts, the design adopts 6-way step motor control module, 1-way DC motor driving module, 1-way relay control module, and 1-way LCD driving module.

(3) Exchange Channel Configurations. The control system was configured with 2-way stock processor communications and 2 -way scion processor communications.

4.3. Design of Control System Software. Control system software includes two parts which are rootstock processing control software and scion processing control software.

4.3.1. Design of Main Control Module for Rootstock Processor. The functionality of the rootstock main control module is to implement stock processing, which includes stock robot hand clipping, stock moving, stock inversed " $\Omega$ " shaped cutting, and sapling binding. The flow diagram of rootstock control software is shown in Figure 12.

4.3.2. Design of Main Control Module for Scion Processor. The functionality of the scion processor main control module is to implement scion processing, including LCD display, scion robot hand clipping, scion moving, and " $\Omega$ " shaped cutting. The program diagram is similar to that of the rootstock processing main control module program. The flow diagram of scion control software is shown in Figure 13.

\section{Experiment}

The developed sapling grafting robot system is shown in Figure 14. Grafting experiments have been carried out in a lab environment.

\subsection{Experimental Objects and Evaluation Criteria}

5.1.1. Experimental Objects. The grafting experiment adopted straight poplar branches that have diameter of about $4 \sim 6 \mathrm{~mm}$ and length around $100 \mathrm{~mm}$. Totally, 100 rootstock samples 


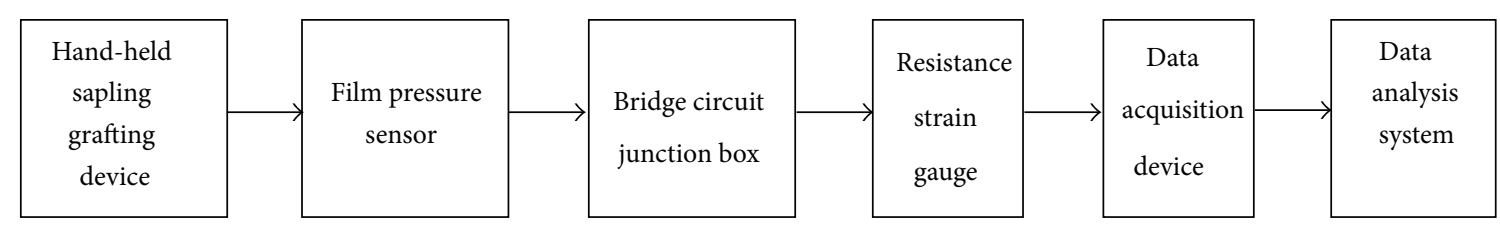

FIgURE 9: Flow diagram of the test circuit.

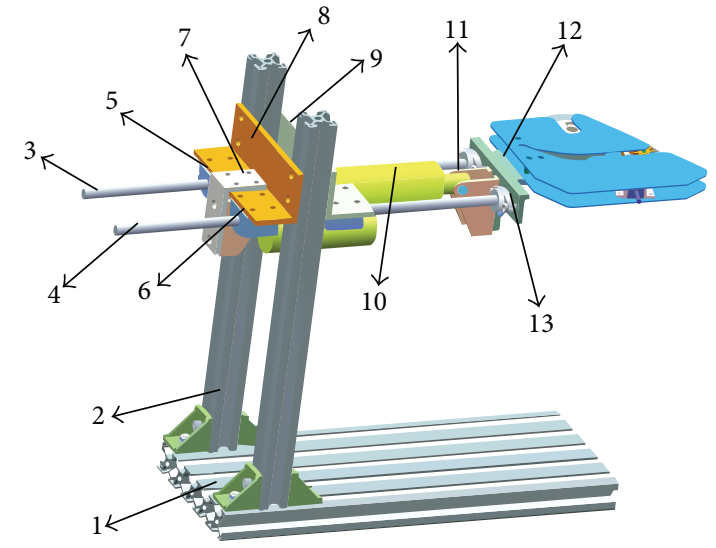

(a)

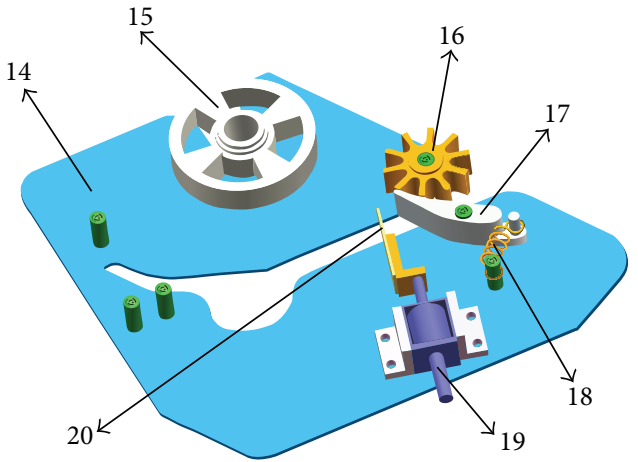

(b)
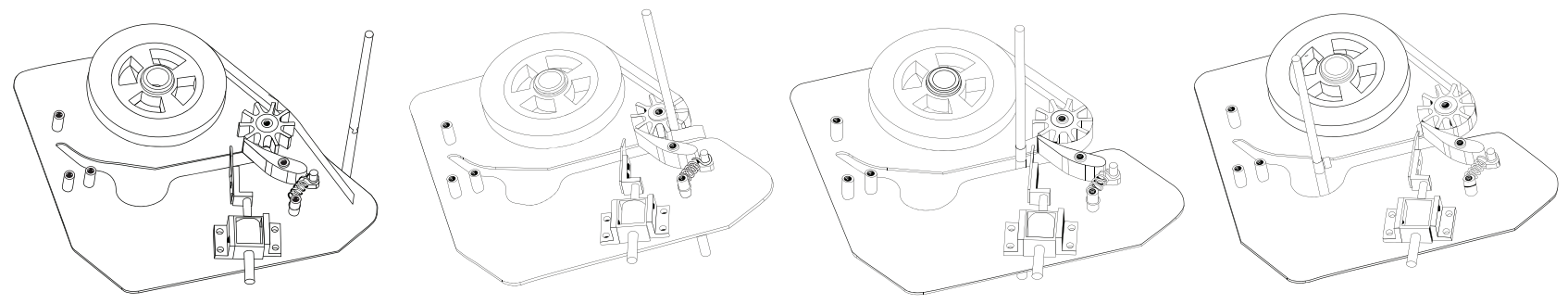

(c)

FIGURE 10: Structure of the binding mechanism. (a) Binding mechanism integrated diagram. (b) Internal diagram of the binding components. (c) Working process. 1: base board, 2: vertical pillar, 3: guiding shaft I, 4: guiding shaft II, 5: sliding unit I, 6: sliding unit II, 7: push rod mounting base, 8: left mounting base, 9: right mounting base, 10: electrical push rod, 11: guiding shaft base I, 12: moving base, 13: guiding shaft base II, 14: cover board, 15: belt wheel, 16: gear, 17: claw, 18: spring, 19: electromagnet, and 20: cutting blade.

and 100 scion samples are chosen, paired according to diameter and then divided into 10 groups.

\subsubsection{Grafting Evaluation Criteria}

(1) The criteria for successful rootstock cutting include inverted $\Omega$ shaped cutting cross section, giving smooth surfaces without burr.

(2) The criteria for successful scion cutting include normal $\Omega$ shaped cutting cross section, giving smooth surfaces without burr.

(3) The criteria for successful grafting include (a) good binding between rootstock and scion; that is, the two central lines are aligned, in good contact without deflections, (b) the rootstock and scion are basically correctly bound; that is, the two central lines are aligned but the contact may not be in perfect condition or there are deflections, and (c) the rootstock and scion are badly bound including bad alignment, failed contact, and deflections. Among these, both (a) and (b) are regarded as successful grafting.

(4) The criteria for successful binding: the binding belt tightly wraps the grafting saplings, without obvious misplacement of the saplings.

5.2. Experimental Results and Analysis. Based on the abovementioned assessment criteria, experiments have been conducted using the developed grafting robot. Among the sapling success statistical data, $43.3 \%$ are good, $44 \%$ are acceptable, and $12.7 \%$ are poor. Therefore, the grafting efficiency is $87.3 \%$.

The results of stock cutting and scion cutting and binding success rate are shown in Table 1, and corresponding curves are shown in Figure 15. Experiments show that the stock cutting efficiency is $98.4 \%$, the scion cutting efficiency is $98.9 \%$, and the binding efficiency is $68.9 \%$. 


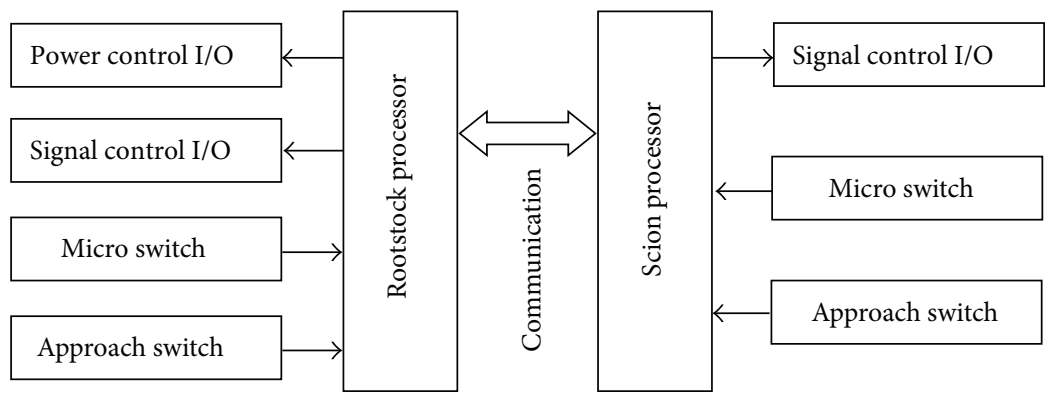

Figure 11: Structure of the control system.

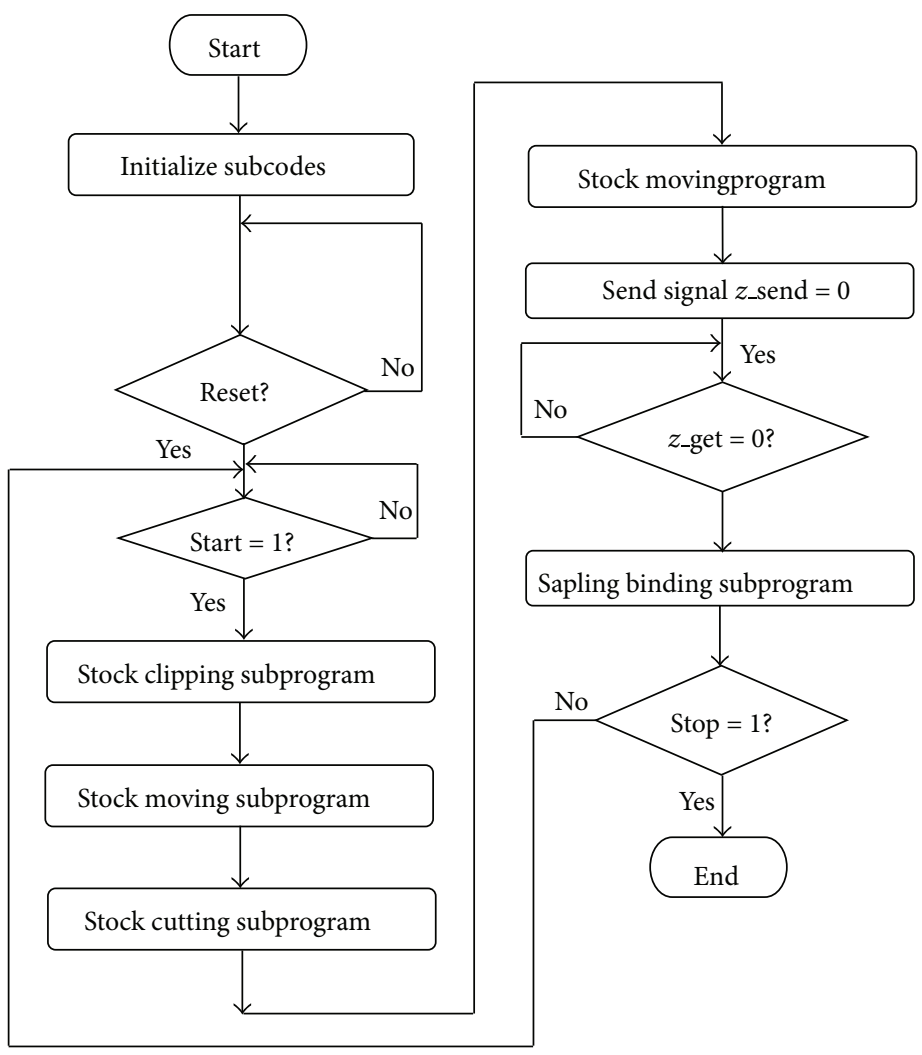

FIGURE 12: Flow diagram of stock processing main control module.

TABLE 1: Results of stock cutting, scion cutting, and binding experiments.

\begin{tabular}{lccc}
\hline Group & Stock cutting success rate (\%) & Scion cutting success rate (\%) & Binding success rate (\%) \\
\hline 1 & 100 & 99 & 63 \\
2 & 98 & 98 & 71 \\
3 & 99 & 100 & 76 \\
4 & 98 & 99 & 71 \\
5 & 99 & 98 & 73 \\
6 & 98 & 100 & 73 \\
7 & 98 & 98 & 70 \\
8 & 97 & 99 & 62 \\
9 & 98 & 99 & 61 \\
10 & 99 & 99 & 69 \\
\hline Total & 98.4 & 98.9 & 68.9 \\
\hline
\end{tabular}




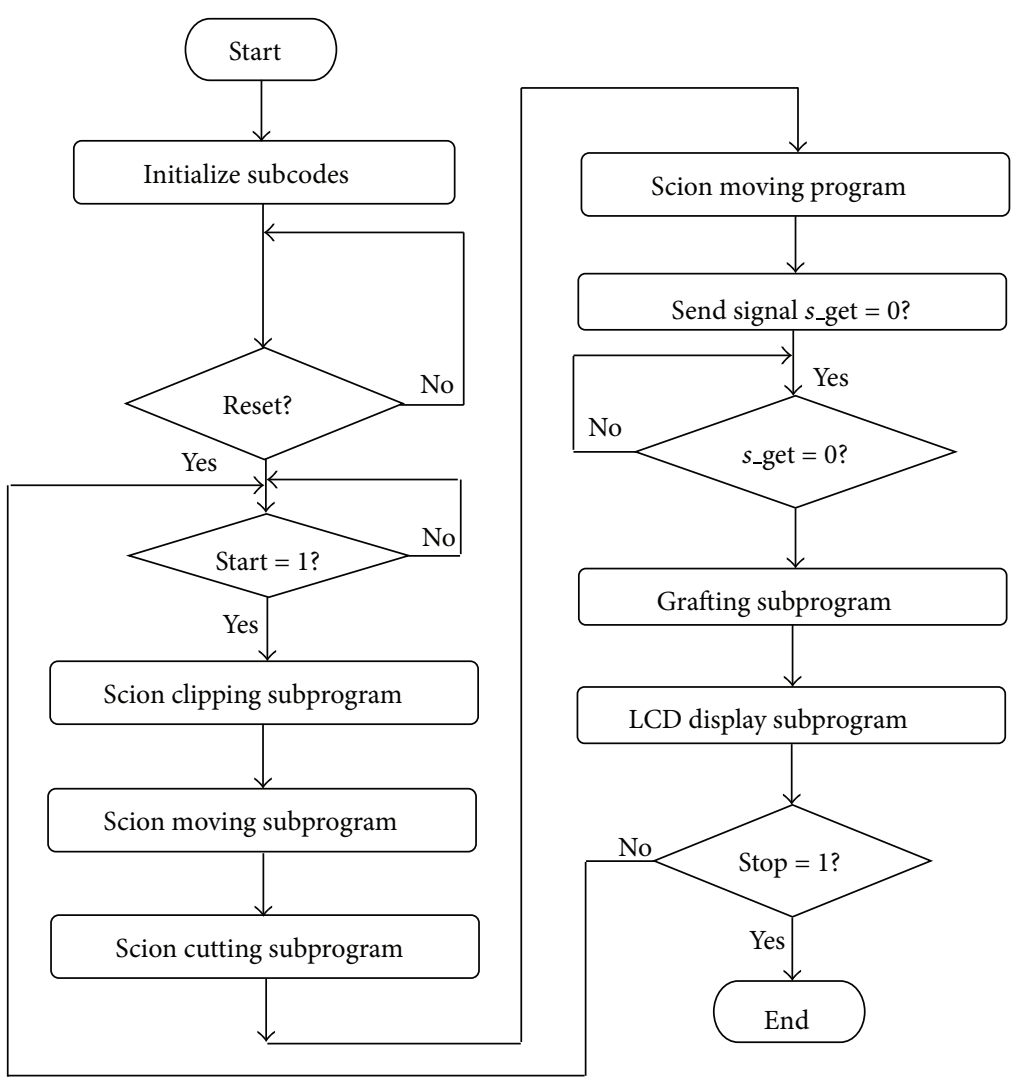

FIGURE 13: Flow diagram of scion processing main control module.

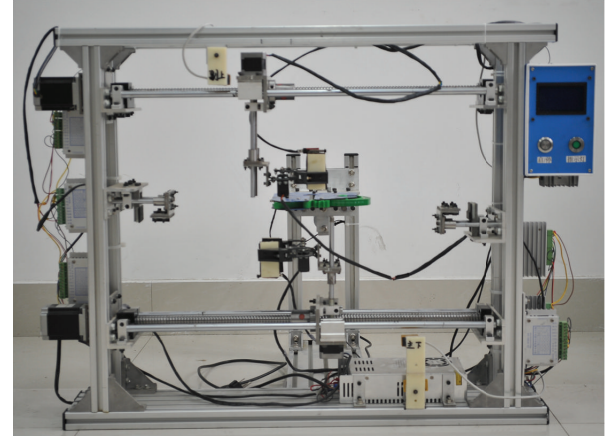

FIgURE 14: The developed sapling grafting robot.

The results of consumption time for sapling grafting experiments are shown in Table 2, and corresponding curves are shown in Figure 16. Experiments show that the average time for stock cutting is 2.20 seconds, the average time for scion cutting is 2.18 seconds, and the average time for one grafting is 34.40 seconds.

The experimental results indicate that the cutting success rates for rootstocks and scions are high, but due to individual differences between stock and scion samples, errors exist in the alignment of the cutting surfaces when binding them together, which affects the grafting success rates. When there are bends near the sapling joining location, the sapling binding success rates decrease. Stock cutting, scion cutting,

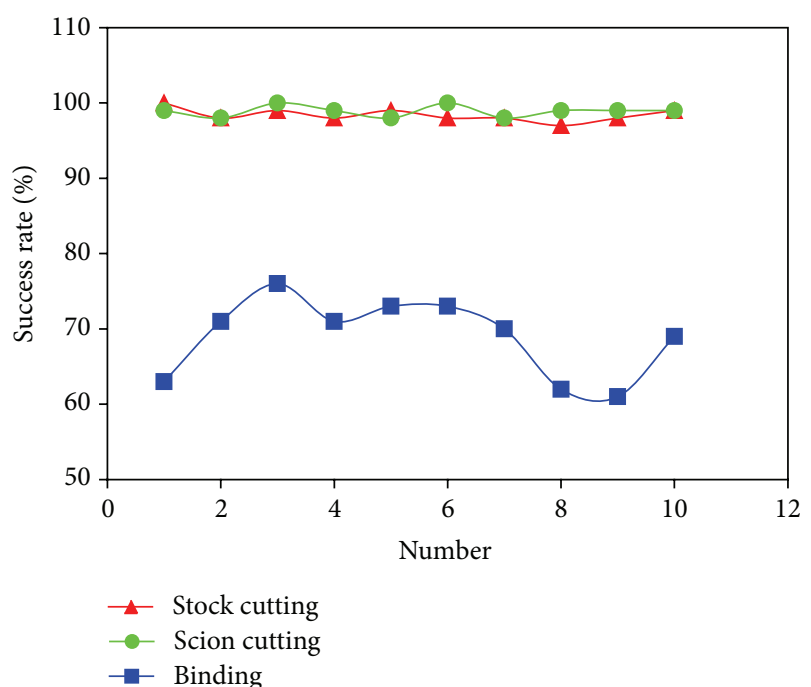

FIGURE 15: Curves of stock cutting, scion cutting, and binding success rate.

and sapling binding processes take relatively short time and the efficiencies are high. However, due to the large empty strokes of the main moving parts, the overall grafting process has a relatively low efficiency.

Further studies include automatically pairing rootstock and scion samples, automatically aligning rootstock and scion 
TABLE 2: Results of consumption time for sapling grafting experiments.

\begin{tabular}{lccc}
\hline Group & Stock cutting average time $(\mathrm{S})$ & Scion cutting average time $(\mathrm{S})$ & Average grafting overall time (S) \\
\hline 1 & 2.2 & 2.1 & 33 \\
2 & 2.1 & 2.2 & 35 \\
3 & 2.0 & 2.0 & 32 \\
4 & 2.2 & 2.2 & 39 \\
5 & 2.2 & 2.3 & 35 \\
6 & 2.3 & 2.2 & 34 \\
7 & 2.1 & 2.1 & 36 \\
8 & 2.3 & 2.2 & 31 \\
9 & 2.2 & 2.3 & 33 \\
10 & 2.4 & 2.2 & 36 \\
\hline Total & 2.20 & 2.18 & 34.40 \\
\hline
\end{tabular}

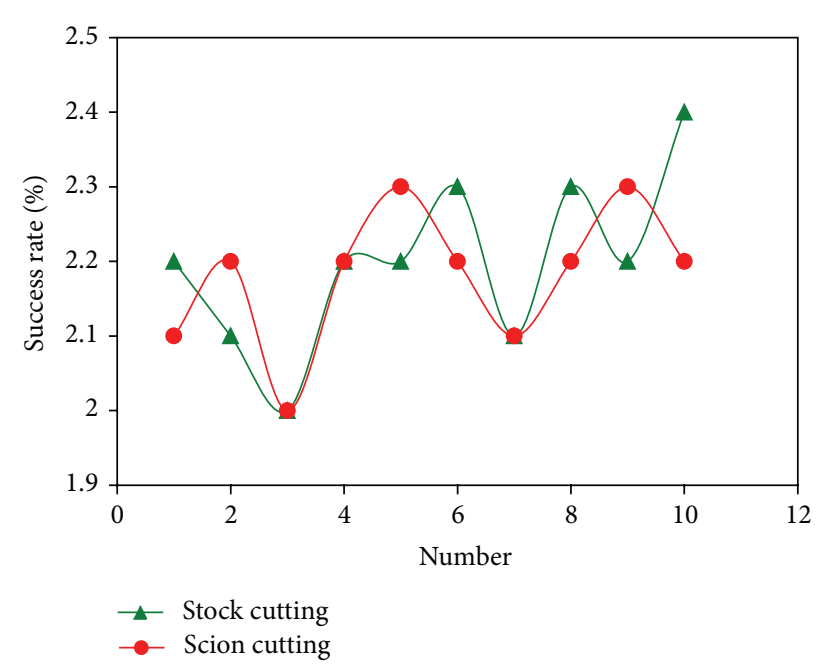

FIGURE 16: Curves of consumption time for stock cutting and scion cutting.

cutting surfaces, accurately wrapping saplings, automatically detecting grafting sapling qualities, and automatically detecting the accuracy of moving parts' motions. The main structural dimensions should also be further optimized.

\section{Conclusion}

(1) An automatic sapling hard branch grafting robot has been developed, which can implement clipping, moving, positioning, cutting, binding, and wrapping rootstock and scion saplings.

(2) Experimental results indicate that the grafting success rate of this robot is $87.3 \%$ and the binding success rate is $68.9 \%$.

\section{Conflict of Interests}

The authors declare that there is no conflict of interests regarding the publication of this paper.

\section{Acknowledgments}

This work was supported by the Natural Science Foundation of Shandong Province, China (Grant nos. ZR2011EL038 and ZR2012CQ026), and A Project of Shandong Province Higher Educational Science and Technology Program (Grant nos. J11LD16 and J12LB63).

\section{References}

[1] Y. Nishiura, N. Honami, and T. Taira, "Development of a new grafting method (part 4)-robotization of grafting operation," Journal of the Japanese Society of Agricultural Machinery, vol. 61, no. 6, pp. 103-112, 1999.

[2] Y. Osamu and F. Akiko, "Growth adjustment technique of seedling optimal for grafting by seedling storage for cucumber full-automatic machine grafting," Tokyo Agricultural Research, vol. 55, no. 1, pp. 201-202, 2002.

[3] K. Ken, S. Kenta, and S. Sadao, "Study on automation of seedlings feeding for grafting robot for cucurbitaceous vegetables (part1)-necessary accuracy for cotyledon orienting mechanism," Journal of the Japanese Society of Agricultural Machinery, vol. 68, no. 6, pp. 117-123, 2006.

[4] C.-H. Kang, G.-S. Han, T.-H. Noh et al., "Splice grafting robot for fruit and vegetable plants: World Intellectual Property Organization," WO/2005/089532, 2005.

[5] S. Chen, Y. C. Chiu, and Y. C. Chang, "Development of a TubingGrafting robotic system for fruit-bearing vegetable seedlings," Applied Engineering in Agriculture, vol. 26, no. 4, pp. 707-714, 2010.

[6] J. M. Lee, C. Kubota, S. J. Tsao et al., "Current status of vegetable grafting: diffusion, grafting techniques, automation," Scientia Horticulturae, vol. 127, no. 2, pp. 93-105, 2010.

[7] Y.-C. Chang, S. Chen, Y.-C. Chiu, L.-H. Lin, and Y.-S. Chang, "Growth and union acclimation process of sweet pepper grafted by a tubing-grafting robotic system," Horticulture Environment and Biotechnology, vol. 53, no. 2, pp. 93-101, 2012.

[8] Z. Libin, Y. Qinghua, B. Guanjun et al., "Overview of research on agricultural robots in China," International Journal of Agricultural and Biological Engineering, vol. 1, no. 1, pp. 12-21, 2008.

[9] C. Kubota, M. A. McClure, N. Kokalis-Burelle, M. G. Bausher, and E. N. Rosskopf, "Vegetable grafting: history, use, and current technology status in North America," HortScience, vol. 43 , no. 6 , pp. $1664-1669,2008$. 
[10] M. Li, C. Tang, F. Xie, M. Wu, and S. Sun, "Experimental study on mechanical properties of peach seedling," Transactions e of the Chinese Society of Agricultural Engineering, vol. 21, no. 3, pp. 29-33, 2005.

[11] Y. Zhao, T. Zhang, and L. Yang, "An experimental study on cutting resistance of root stock of branch to branch graft," Agricultural Mechanization Research, vol. 30, no. 7, pp. 146-148, 2008.

[12] M. Li, S. Dai, C. Tang, and Y. Xiang, "Simulation test on plantletcutting mechanism of grafting robot," Transactions of the Chinese Society of Agricultural Engineering, vol. 24, no. 6, pp. 129-132, 2008.

[13] Y. Zhao, T. Zhang, and H. Wang, "Cutting mechanism of root parental stock in automatic sapling grafting machine," Transactions of the Chinese Society of Agricultural Engineering, vol. 24, no. 9, pp. 79-83, 2008.

[14] X. Jiang, "Design of model PJJ-50 grape grafting machine," Agricultural Equipment \& Vehicle Engineering, vol. 11, pp. 7-9, 2011.

[15] F. Wang, M. Liu, X. Wu et al., "Study and design of grafting robot for Camellia oleifera seedlings," Forest Machine and Equipment, vol. 39, no. 4, pp. 36-39, 2011.

[16] Q. Sun, D. Zhao, and Y. Zhao, "Mechanical design and test for a sapling grafting robot based on the Cleft method," Journal of Henan Technology University, vol. 34, no. 1, pp. 69-71, 2013. 

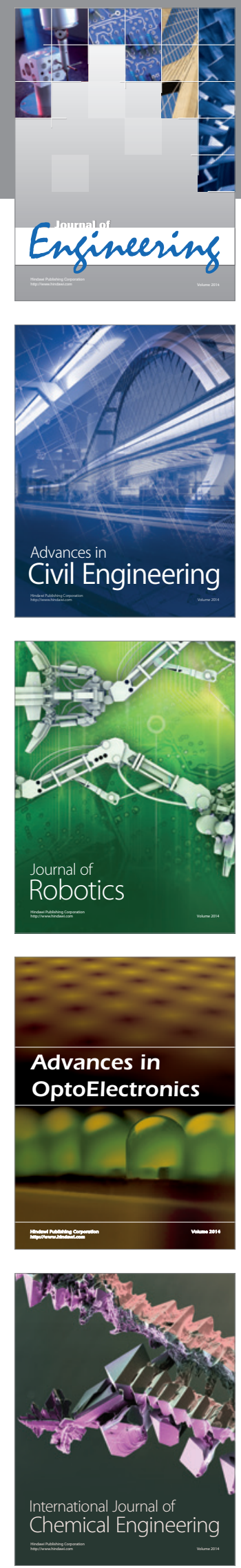

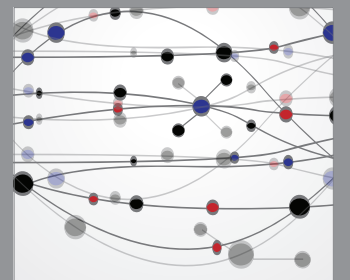

The Scientific World Journal
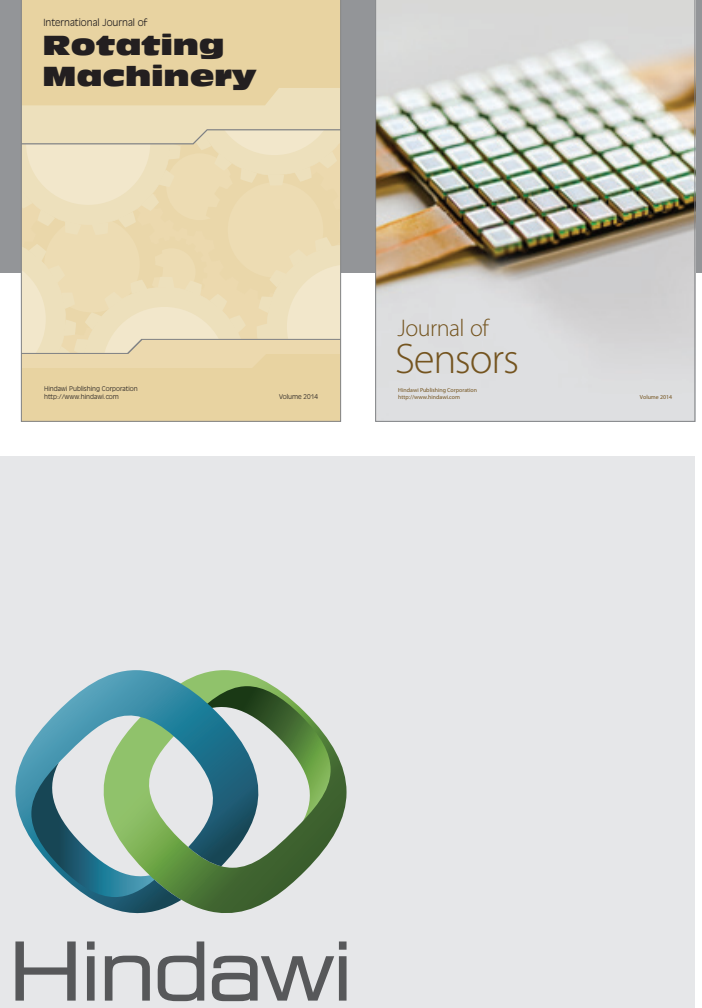

Submit your manuscripts at http://www.hindawi.com
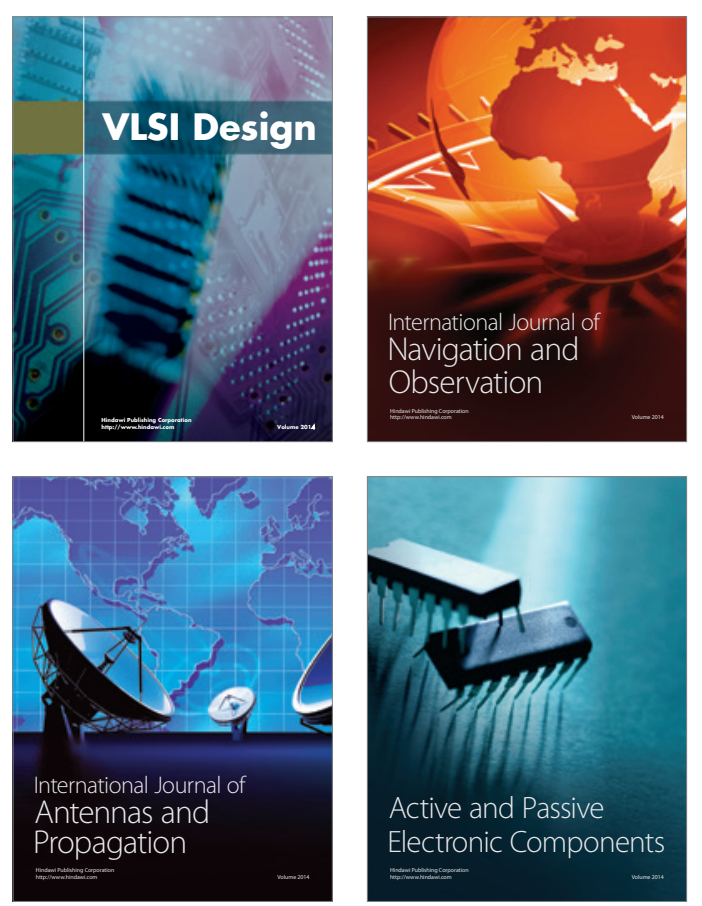
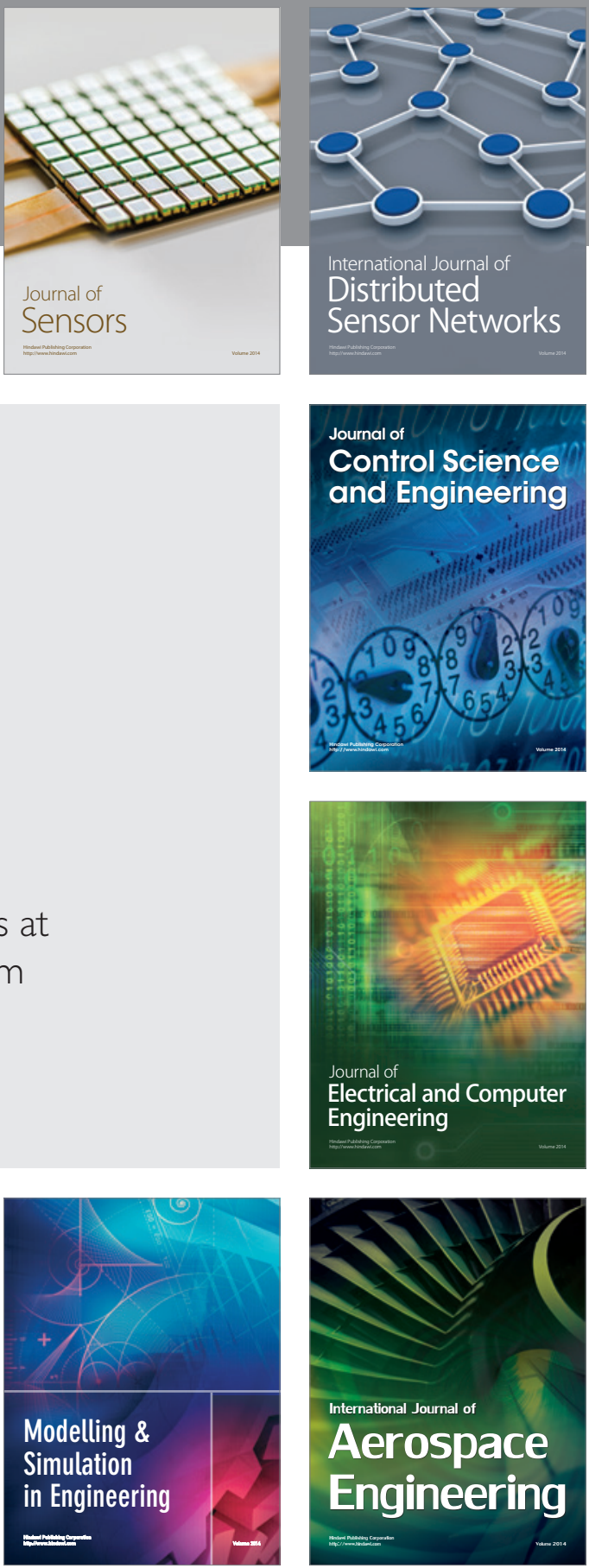

Journal of

Control Science

and Engineering
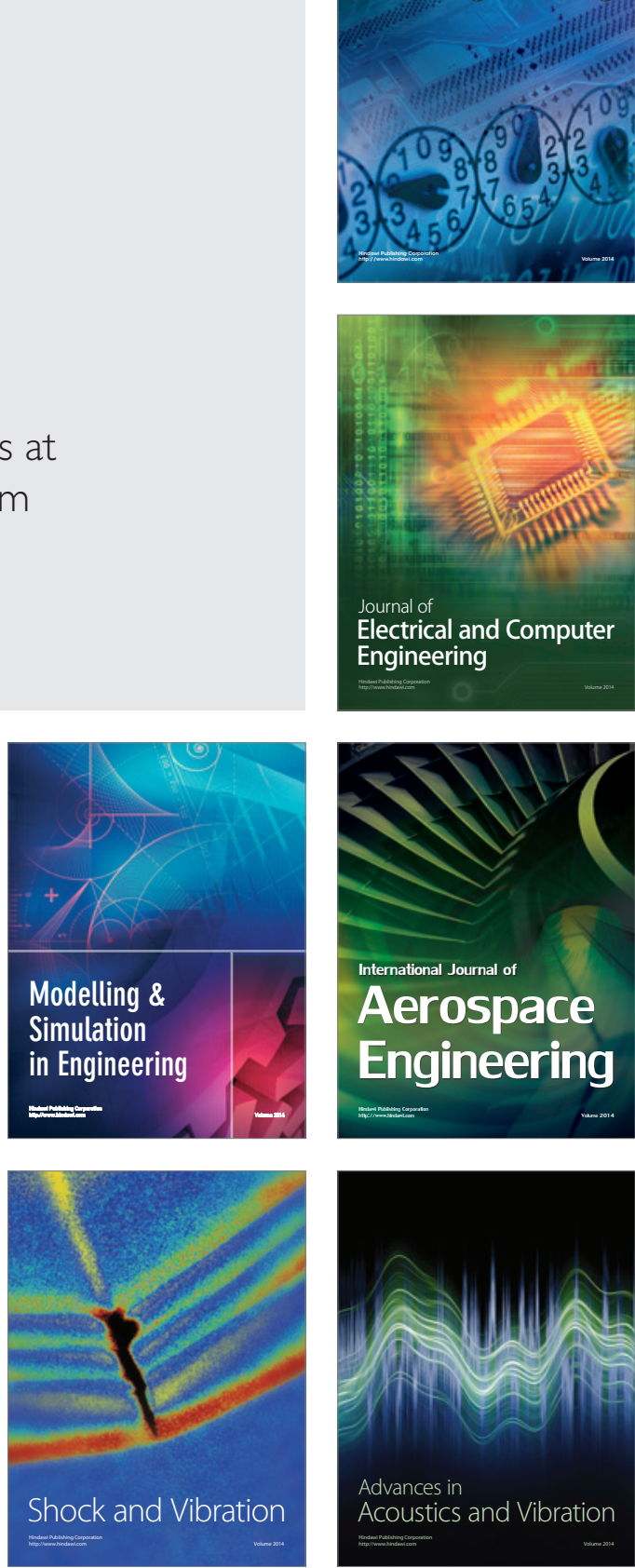